\title{
Ponencias: Evaluación en la educación superior
}

\section{La autoevaluación-autorregulación y la excelencia de la educación superior: Una experiencia valiosa}

\author{
Ana Cecilia Arias Quirós \\ Olga Echeverría Murray
}

\begin{abstract}
Resumen: El trabajo deriva de la experiencia que durante cinco años hemos venido acumulando en la Escuela de Antropología y Sociología de la Universidad de Costa Rica, alrededor de un proceso de autoevaluación-autorregulación que puede convertirse en un camino, para lograr la excelencia académica en el marco de una Universidad Estatal, en este caso concreto, la Universidad de Costa Rica.

Sin duda alguna, las condiciones sociales y culturales que enfrentamos actualmente nos plantean un reto en lo que se refiere a la formación académico-profesional de los científicos sociales, particularmente de los antropólogos y antropólogas, sociólogos y sociólogas. Una tensión permanente y acentuada entro lo local y lo global, entre lo particular y lo general, una lucha continua entro la promoción del desarrollo y preservación de lo identitario, un mundo cada vez más comunicado, más tecnificado, pero de igual forma, cada vez más pobre, que afecta hasta su desaparición a la naturaleza. Un mundo que exige mayor preparación a los profesionales, actualización permanente, eficiencia en su quehacer, una preparación académica y un perfil académico-profesional dispuesto para dar respuesta a todos estos requerimientos. Los procesos de autoevaluación-autorregulación permiten -llevarlos adecuadamente- a las unidades académicas, basados en el diálogo y el análisis integral del quehacer docente-administrativo, definir nuevos y posibles escenarios, una formación académica y profesional pertinente, viable, reflexiva y comprometida, especializada y actualizada tecnológicamente hablando y humanista.
\end{abstract}

Las transformaciones que vivimos actualmente producto de los procesos de globalización, inciden en cambios de índole económico, social, político, ecológico, institucional y en la educación formal. Imponen, un nuevo orden al modificarse la producción, el comercio, la tecnología; provocan la participación asimétrica de los actores sociales y graves contradicciones en la vida diaria que se reflejan en los procesos de enseñanza-aprendizaje.

Expresiones cotidianas de esas transformaciones, son: los procesos de migración que traen consigo la aparición de sociedades multiétnicas, donde muchas veces se instaura la violencia, la discriminación y la xenofobia. La pobreza al interior de los países, y la relación desigual entre países pobres y aquellos más industrializados. La formación de grandes bloques regionales que luchan por su hegemonía y tratan de imponer la homogeneización, generando tensiones permanentes entre lo local y lo global, provocando la transculturación, el desarraigo de costumbres, esquemas de valores, creencias, crisis identitarias, o, a la inversa, la reafirmación de identidades que hacen aflorar profundos conflictos, al no reconocerse el carácter multicultural de esos pueblos y al no respetarse la existencia de modos de vida diversos y disímiles. 
La destrucción de la naturaleza, con la consabida degradación del ambiente, el embate contra complejos ecosistemas producto de la biodiversidad existente en nuestro planeta; la destrucción de sitios arqueológicos, lo cual limita el conocimiento e interpretación de nuestra historia precolombina, son algunos ejemplos, de la necesidad de conocer, profundizar en estas transformaciones actuales y comprender su incidencia e influencia en los centros de educación superior.

Lo anterior provoca retos que necesitamos, enfrentar pues se relacionan profundamente con la misión de una universidad como la Universidad de Costa Rica y por ende con disciplinas como la Antropología, la Arqueología y la Sociología.

La tradición científica, por ejemplo, de la Antropología, como se enuncia en la fundamentación y justificación de nuestro plan de estudios parte de

“...una visión holística y diacrónica del quehacer humano y abocada al estudio de la diversidad cultural, de la relación ser humano-naturaleza, está llamada a ofrecer aportes relevantes a la identificación de nuevas vías alternativas de transformación social, que pongan énfasis en la relación humana, la diversidad, el pluralismo, y la participación real de hombres y mujeres en la construcción de su proyecto futuro y el de las generaciones venideras" (Resolución No. VD6264-97, pág. 1).

En el caso de la Sociología, en la presentación del nuevo plan de estudios se enfatiza que

"una de las concepciones vertebrales, es que la sociología, durante el inicio del nuevo milenio, debe ser visualizada como un saber humanístico, consustancial a la formación de todos los profesionales del país, a la vez que el sociólogo o socióloga debe ser un profesional que busque su integración investigativa y activa en equipos interdisciplinarios". (Resolución No. VD6264-97, pág. 1).

Estas fundamentaciones y justificaciones demuestran que el rumbo de la ense- ñanza y la práctica de las tres disciplinas, deben preparar al estudiante para "utilizar su conocimiento teórico y el instrumental técnico en el acercamiento a la realidad y a la información necesaria para comprender la diversidad y regularidad de los hechos sociales" (Salom y otros: 1993, p. 68).

Las tres disciplinas deben jugar un papel importante para desarrollar conocimientos que puedan contribuir a estrechar vínculos entre los pueblos, especialmente de la región centroamericana, a profundizar en el conocimiento y reconocimiento de las múltiples identidades étnicas y nacionales, a abordar las dinámicas micro y macro-sociales, tal y como se establece en nuestros planes de estudio. "Profundizar y sistematizar el conocimiento cultural desde la perspectiva teórico-metodológica, como desde su aplicación a la realidad imperante en cada momento histórico de la sociedad costarricense". (Resolución No. VD6264-97, pág. 9).

Queremos formar estudiantes, en el caso de la Antropología, con un perfil académico-profesional que les permita: participar en investigaciones con una visión comprensiva sobre la relación del ser humano con su medio social, cultural y ambiental; comprender holística e históricamente la diversidad cultural y socioeconómica y la forma en que ésta condiciona la vida en sociedad; analizar la forma en que intervienen los factores biológicos y culturales en la determinación de la conducta humana; analizar los universos simbólicos; intervenir en equipos de trabajo inter y transdisciplinarios; participar en la formulación y ejecución de propuestas de transformación social y cultural, especialmente en contextos locales y microsociales, sólo para enumerar algunas de las responsabilidades. Y en el caso de la Sociología, ésta

"no solamente debe ser muy funcional en su desempeño profesional, sino que debe demostrarlo en la práctica. Para ello se propone un plan de estudios mediante el cual se estimule su contacto con los diferentes niveles 
de la realidad social, y se posibilite la articulación entre la teoría, la práctica y dicha realidad. Para ello, el sociólogo deberá adquirir una sólida formación personal y humanística, para el trabajo interdisciplinario y multidisciplinario, así como técnicas diversas, junto con valores éticos sobre su papel y su imagen socio-profesional, sobre la necesidad del auto-aprendizaje constante y la superación personal y profesional”. (Resolución No. VD6264-97, pág. 11-12).

Basándonos en lo expuesto anteriormente, tenemos al frente el reto de cumplir con una función formativa en donde es prioritaria la búsqueda crítica del conocimiento y del aprendizaje; el fomento del pensamiento analítico y creativo; la formación ética de los educandos, todo ello en consonancia y de acuerdo a nuestras tradiciones culturales, creencias, valores, con el fin de llevar a cabo un proceso de enseñanza-aprendizaje pertinente.

Es en este contexto que un proceso de autoevaluación-autorregulación es valioso, ya que se caracteriza fundamentalmente por:

- La participación voluntaria de las unidades académicas facultades $\mathrm{u}$ otras instancias universitarias.

- La participación de todos los actores sociales de los diferentes sectores: docente, administrativo y estudiantil.

- Un ambiente de diálogo abierto, franco, dinámico, sistemático, riguroso y transparente.

- Un ambiente crítico y constructivo, propositivo.

- Interno e independientemente en cada unidad académica.

Los principios orientadores de un proceso de esta índole deben abordarse según las características de la unidad "autoevaluada", pues cada una tiene sus respectivos retos, cada una requiere privilegiar distintos atributos, habilidades, conocimientos entre sus profesores y estudiantes. El cambio dentro de cada unidad, está asociado con desafíos, muy particulares, que cada una tenga y los procesos de aprendizaje pueden tomar o necesitan tomar muy diferentes formas, tal y como lo establecen los distintos planes de estudio. En nuestro caso concreto el desafío es: "promover permanentemente la reflexión, evaluación y transformación de los planes de estudio para que puedan responder a los requerimientos y circunstancias propias de los tiempos actuales" (Resolución No. VD-6264-97, pág. 9).

Un proceso de autoevaluación-autorregulación, nos permite conocer las fortalezas, problemas y limitaciones que tenemos como unidades o facultades, los desafíos “internos" y "externos" para posteriormente "autorregularnos" o sea, planificar la gestión, basándonos en procedimientos bien definidos y fundamentados, no solo desde el punto de vista de la administración, sino en cuanto a la implementación de los planes de estudio, programas de los cursos y técnicas de enseñanza versátiles y diversas, con el objetivo de lograr una formación teóricometodológica, que refuerce la dimensión integral y humanista, la conciencia social de docentes y educandos, tal y como lo señalan nuestros planes de estudios y el Estatuto Orgánico de la Universidad de Costa Rica.

Es nuestro interés socializar algunos de los resultados que obtuvimos en el proceso que iniciamos en 1995 y que por su misma naturaleza aún permanecen a través de la experiencia de autorregulación. Nos motiva compartirlo pues lo conceptualizamos como una actividad productiva y pertinente en la coyuntura actual para cualquier Facultad, Unidad Académica, e incluso para instancias administrativas de la Universidad.

En el caso concreto de la Escuela de Antropología y Sociología esta experiencia fue muy valiosa desde sus inicios al poder: 
Trabajar conjuntamente con los miembros de otras unidades participantes como la Escuela de Química, la Facultad de Farmacia, la Sede Regional del Atlántico (Turrrialba) y los miembros del Centro de Evaluación Académica (CEA), elaborando las dimensiones de análisis en las áreas y sectores que las diferentes unidades consideraban importantes de evaluar, así como definiendo los criterios para ello.

Revisar los resultados de las evaluaciones de los cursos que se habían hecho desde 1993 al 1996, los resultados de los Talleres de Reflexión Académica, de Investigación y Acción Social, de Reforma Currricular, sobre el Perfil Profesional y su Contexto, entre otras actividades que se habían hecho, en el marco de la revisión de los planes de estudio.

Elaborar, por parte de la comisión encargada, los instrumentos para recabar información de cada uno de los sectores a evaluar, que fueron luego revisados y analizados con los especialistas del área de métodos y técnicas y validados en grupos focales para su correspondiente corrección.

- Aplicar los instrumentos a los diferentes sectores, el análisis posterior de la información, lo cual fue una experiencia de formación teórico metodológica y de carácter formativo, de un valor incalculable.

La retroalimentación individual y colectiva entre todos los integrantes de la Escuela (administrativos, docentes y estudiantes), al socializarce la información por medio de varios talleres, otras actividades colectivas y en forma individual, al recibir cada docente, por ejemplo, los resultados de los ítems evaluados en cada uno de sus cursos.

- $\quad$ El diagnóstico exhaustivo que fue posible obtener de las fortalezas y problemas detectados, lo cual permitió hacer énfasis en las áreas de debilidad sobre las cuales sería necesario tomar acciones reguladoras, entendiéndose como áreas de debilidad aquellas en las que algún sector de la unidad consideraba que se debía fortalecer o mejorar en algún sentido.

Asignar a cada una de esas debilidades acciones correctivas, responsables, plazos y recursos necesarios para llevar a cabo los planes de acción.

La repercusión, por la visión holística e integral que se pudo obtener, a partir del proceso, es posible visualizarla por medio de lo que se consignó como prioritario, aplicando índices de importancia, por ejemplo: en el sector administrativo se priorizaron seis debilidades, la más importante el ambiente laboral: por el recargo de tareas, confusión de funciones, desconocimiento de la vocación y responsabilidades acerca de la Universidad y el esquema compartimentado del sector administrativo. Para solucionar esta debilidad se mencionaron 6 acciones diferentes a realizar a mediano plazo, tales como:

"desarrollar talleres de capacitación y reflexión sobre la misión y la vocación de la Universidad de Costa Rica, de la Facultad de Ciencias Sociales y de la Escuela de Antropología y Sociología, con participación de personal administrativo, estudiantes y docentes". (Araya y otros: 1998 tomo sector administrativo).

Sector docente: la práctica docente recibió el puntaje más alto por inadecuadas técnicas didácticas utilizadas por los profesores, predominio de la figura del profesor, limitaciones en práctica docente, necesidad de actualización teórica; dos acciones a corto plazo se debían realizar 
frente a esta debilidad, una de ellas: "organizar actividades de capacitación y actualización, para docentes, sobre técnicas didácticas dinámicas y participativas, así como sobre relaciones personales". (Araya y otros: 1998 tomo sector docente).

Sector estudiantil: el rendimiento estudiantil era la debilidad más fuerte por la falta de responsabilidad en el cumplimiento de obligaciones, estudio de la materia, lecturas, asistencia y participación; poca dedicación a la carrera y sobrecarga académica. Aquí se definieron 4 acciones, por ejemplo: "hacer explícito el perfil del estudiante de ingreso y graduación, de acuerdo con los Planes de estudio y la misión académica de cada departamento" y "realizar un diagnóstico de la situación real, socio-económica y psico-social de la población estudiantil de la Escuela, a partir del cuarto semestre de la carrera". (Araya y otros: 1998 tomo sector estudiantil).

En el área de investigación y acción social se plantearon problemas y propuestas muy concretas, pero el colectivo a raíz de que no había un "registro" o "memoria" de lo que había sido la investigación y la acción social en la Unidad Académica pues los archivos de los últimos años estaban incompletos y no se sabía con exactitud cuáles habían sido los criterios utilizados para evaluar los proyectos presentados, asignar cargas y prórrogas, decidió que el primer paso debía ser "completar el diagnóstico para a muy corto plazo: aportar elementos para la elaboración de una política clara de investigación y acción social y aportar criterios e instrumentos para la evaluación continua de estas dos áreas" (Araya y otros: 1998, tomo Investigación Acción Social).

Todo esto ha generado la ejecución de acciones muy concretas por parte de las instancias de dirección y de los diferentes sectores de la Unidad Académica:

\section{a. A nivel administrativo:}

- Participación en el "Proyecto Auditoría Administrativa" en el que se evaluaron activos fijos, vacaciones, declaraciones juradas de jornadas y horarios, entre otros.

- Reorganización de labores, funciones y responsabilidades de las plazas asignadas al personal administrativo. Creación de archivos documentales informatizados con bases de datos sobre: proyectos de investigación y acción social, expedientes del personal docente y administrativo, acciones de personal, entre otros.

\section{b. En el plano estrictamente aca- démico}

Reforma curricular en la Licenciatura de Sociología, Antropología Social y Arqueología.

- II Encuentro de Sociólogos en octubre de 1998, bajo el título "Conocer y resolver en un mundo en transición: la actualidad del quehacer sociológico". Iniciación de las actividades académicas de la Maestría en Antropología en el I ciclo de 1998.

- Dos talleres de reflexión siguiendo las técnicas "Nominal de Grupo Participativo y Planificación Orientada a Objetivos (ZOPP)" con docentes y estudiantes para detectar problemas en el nuevo plan de estudios, sus causas, efectos y posibles soluciones. Evaluación interna, por iniciativa de los miembros de la Escuela, de todos los cursos impartidos en el I ciclo de 1999. II Congreso Costarricense de Antropología y Arqueología, con el título "Por nuestra identidad y memoria antropológica”, en diciembre de 1999. Talleres de didáctica universitaria a cargo del Departamento de Didáctica de la Escuela de Formación Docente para profesores y profesoras de la Unidad Académica. 
- $\quad$ Profesores invitados y visitantes que han impartido cursos y talleres de actualización y capacitación.

- $\quad$ Apertura de nuevos cursos con contenidos novedosos.

\section{c. A nivel estudiantil}

- Estudios de casos relativos a la relación docente-estudiante; condición crítica y problemáticas individuales de algunos estudiantes, en campos relativos a su condición, socioeconómica y su inserción a la Universidad.

No podemos mencionar todos los campos en los que ha sido posible la "autorregulación" interna, baste con recalcar el nivel de concienciación que genera una actividad de esta índole, al hacer énfasis en las debilidades detectadas en cada sector o área evaluada. Categorizar y priorizar esas debilidades favorece un clima de análisis, de reflexión continua y de búsqueda de soluciones.

Permite mayor claridad en cuanto a las necesidades del contenido curricular destinado a la formación de los futuros profesionales en nuestras disciplinas. Fomenta nuevas metodologías de enseñanza. Ayuda a visualizar un proyecto académico más acorde con los retos actuales y a definir políticas de investigación y acción social que retroalimenten la docencia.

Es un esfuerzo que ayuda a profundizar en los problemas administrativo-docentes, conocimiento fundamental en toda acción dirigida a la actualización y capacitación del personal tanto docente como administrativo. Es una herramienta eficaz para cumplir los objetivos del plan de estudios y el perfil académico profesional de quienes se graduén en el campo de la sociología, la antropología o la arqueología (para citar nuestro caso concreto). Tiene, además, repercusión directa en todo lo relacionado con la vida académica institucional y de gestión universitaria y algo que nos parece central, nos permite valorar las fortalezas múltiples y diversas con que contamos.

Creemos necesario mencionar que, nuestra Institución ha hecho esfuerzos importantes en el campo de la autoevaluación-autorregulación, razón por la cual, sería ideal sistematizar y generalizar la experiencia a diversas instancias como: las Vicerrectorías, oficinas coadyuvantes y otras. Si asumen el reto de autoevaluarse, podrán tener una mayor claridad en el papel que deben jugar en cuanto al apoyo, planificación, incentivos y la responsabilidad que deben asumir para fortalecer la legitimidad de estos procesos.

El soporte institucional es fundamental para el éxito y la cristalización, de actividades de esta índole, incluyendo la etapa de acreditación definida entre otras cosas como "una certificación de la calidad académica de un programa o unidad, o un juicio sobre la consistencia entre los objetivos, los recursos y la gestión de una unidad académica” (Martínez: 1997, p. 80).

La acreditación como carta de presentación, implica serias responsabilidades. Antes de someterse a ella, es necesario, poner en marcha la autorregulación con el objetivo de corregir y revisar algunas de las debilidades y problemas encontrados; tener clara la misión del programa, unidad o facultad; los objetivos definidos de acuerdo a esa misión; el mínimo de instalaciones y recursos adecuados; programas académicos dentro de marcos dinámicos y flexibles de procesos de enseñanzaaprendizaje que fomenten el pensamiento analítico, creativo y crítico.

Recordemos que, en el proceso de acreditación un organismo técnico externo a la institución "garantiza" que un determinado programa, unidad académica o facultad, cumple los criterios de calidad y pertinencia 
definidos por esa entidad acreditadora. Es un mecanismo de "reconocimiento social" difícil de conseguir, si no se tienen resueltos por lo menos algunos, de los requerimientos indispensables para ajustarse a los criterios de los evaluadores.

En cualesquiera de estas etapas, se busca la excelencia tanto en el discurso como en la práctica, en lo individual y en lo colectivo, en lo ordinario y en lo extraordinario, en lo académico y en lo administrativo, en las Unidades Académicas y en la Universidad como una totalidad.

La Universidad de Costa Rica, como institución pública tiene la misión de fomentar la excelencia académica, pero además, formar profesionales comprometidos con la equidad, con la sociedad. Está llamada a ser el ojo crítico y la voz propositiva que junto a otras instituciones, promueva el bien común, el bienestar de todos y todas. La universidad pública debe ser la principal protagonista en la promoción de la democracia en la sociedad costarricense y el pilar en la construcción de un mañana mejor.

Quisiéramos terminar esta ponencia, en este I Simposio Internacional de Pedagogía Universitaria, con esta cita:

"Reflexionar y anticiparse a las necesidades del futuro es tarea enriquecedora e inagotable. Los grandes problemas de la humanidad en estos momentos nos obligan a la búsqueda del conocimiento integrador, no parcial. La llamada globalización nos impone el conocimiento de pueblos, culturas, procesos económicos, problemas ambientales y sobre todo la necesidad de comprensión de los valores fundamentales que permitan la rectificación de errores y la búsqueda de soluciones". (Chen-Apuy, Hilda: 1995).

\section{Referencias bibliográficas}

Araya; Echeverría; Escalante; López. Informe final para los Pares Externos.
Comisión de Autoevaluación-Autorregulación. Escuela de Antropología y Sociología. Facultad de Ciencias Sociales. Universidad de Costa Rica, junio, 1998.

Chen Apuy E., Hilda. "La Universidad de Costa Rica en un mundo Inter.-Independiente". En: Universidad y Desarrollo. A contrapelo de los tiempos. Oficina de Publicaciones de la Universidad de Costa Rica, 1995.

Martínez, Eduardo. "La evaluación de la educación superior”. En: Evaluación y Acreditación Universitaria. Metodologías y experiencias. Martínez, E; Letelier, M. (editores). UNESCO; Organización Universitaria Interamericana-OUI; Universidad de Santiago de Chile-USACH; Editorial Nueva Sociedad, 1997.

Plan de Estudios de la Carrera de Antropología. Ciudad Universitaria Rodrigo Facio. Resolución No. 6264-97. Vicerrectoría de Docencia Escuela de Antropología y Sociología. Departamento de Antropología. Universidad de Costa Rica, 1997.

Plan de Estudios de la Carrera de Sociología. Ciudad Universitaria Rodrigo Facio. Resolución No. 6264-97. Vicerrectoría de Docencia Escuela de Antropología y Sociología. Departamento de Antropología. Universidad de Costa Rica, 1997.

Salom, R; Romero, M.; Herrera, L; Ramírez, M. "Reflexiones acerca del Perfil del sociólogo en una propuesta de reforma curricular". En: Cuadernos de Sociología No. 2. Retos y perspectiva de la Sociología en Costa Rica. Escuela de Antropología y Sociología. Universidad de Costa Rica, noviembre 1993. 